\title{
Prognostic role of time to positivity of blood culture in children with Pseudomonas aeruginosa bacteremia
}

Huiting $X u^{1,2}$, Jie Cheng ${ }^{1,2}$, Qinghong $Y u^{1,2}$, Qingyuan $\mathrm{Li}^{1,2}$, Qian $\mathrm{Yi}^{1,2}$, Siying Luo ${ }^{1,2}$, Yuanyuan $\mathrm{Li}^{3}$, Guangli Zhang ${ }^{3}$, Xiaoyin Tian ${ }^{3}$, Dapeng Cheng ${ }^{4}$ and Zhengxiu Luo ${ }^{3^{*}}$ (D)

\begin{abstract}
Background: Pseudomonas aeruginosa (P. aeruginosa) is a major Gram-negative pathogen, which has been reported to result in high mortality. We aim to investigate the prognostic value and optimum cut-off point of time-to-positivity (TTP) of blood culture in children with $P$. aeruginosa bacteremia.

Methods: From August 2014 to November 2018, we enrolled the inpatients with P. aeruginosa bacteremia in a 1500bed tertiary teaching hospital in Chongqing, China retrospectively. Receiver operating characteristic (ROC) analysis was used to determine the optimum cut-off point of TTP, and logistic regression were employed to explore the risk factors for in-hospital mortality and septic shock.

Results: Totally, 52 children with P. aeruginosa bacteremia were enrolled. The standard cut-off point of TTP was $18 \mathrm{~h}$. Early TTP ( $\leq 18 \mathrm{~h}$ ) group patients had remarkably higher in-hospital mortality (42.9\% vs $9.7 \%, P=0.014)$, higher incidence of septic shock ( $52.4 \%$ vs $12.9 \%, P=0.06)$, higher Pitt bacteremia scores [3.00 (1.00-5.00) vs $1.00(1.00-4.00)$, $P=0.046]$ and more intensive care unit admission (61.9\% vs $22.6 \%, P=0.008)$ when compared with late TTP (> $18 \mathrm{~h})$ groups. Multivariate analysis indicated TTP $\leq 18 \mathrm{~h}$, Pitt bacteremia scores $\geq 4$ were the independent risk factors for inhospital mortality (OR 5.88, 95\%Cl 1.21-21.96, $P=0.035$; OR 4.95, 95\%Cl 1.26-27.50, $P=0.024$; respectively). The independent risk factors for septic shock were as follows: TTP $\leq 18 \mathrm{~h}$, Pitt bacteremia scores $\geq 4$ and hypoalbuminemia (OR 6.30, 95\%Cl 1.18-33.77, $P=0.032$; OR 8.15, 95\%Cl 1.15-42.43, $P=0.014 ;$ OR 6.46, 95\% Cl 1.19-33.19 $P=0.031$; respectively).
\end{abstract}

Conclusions: Early TTP ( $\leq 18$ hours) appeared to be associated with worse outcomes for P. aeruginosa bacteremia children.

Keywords: Pseudomonas aeruginosa, Time to positivity, Bacteremia, Children, Outcomes

\section{Intrudoction}

Pseudomonas aeruginosa (P. aeruginosa) is responsible for infections with different starting point sites [1]. Poor outcomes usually occurred in critically ill patients infected with $P$. aeruginosa [2]. Early assessment of the

\footnotetext{
* Correspondence: luozhengxiu816@hospital.cqmu.edu.cn

${ }^{3}$ Department of Respiratory Medicine, Children's Hospital of Chongqing Medical University, Chongqing 401122, China

Full list of author information is available at the end of the article
}

severity of $P$. aeruginosa bacteremia patients may contribute to assisting the therapy and monitor, so as to improve the outcomes of these patients [3, 4]. Some studies have investigated tools to identify patients at high risk of mortality, such as APACHE scores and PRIS $M$ scores [5, 6]. However, the process of these prognostic scores is complex, which leading to inconvenient in clinical work. Therefore, simpler and easier measurement tools are needed in clinical work.

C C The Author(s). 2020 Open Access This article is licensed under a Creative Commons Attribution 4.0 International License, which permits use, sharing, adaptation, distribution and reproduction in any medium or format, as long as you give appropriate credit to the original author(s) and the source, provide a link to the Creative Commons licence, and indicate if changes were made. The images or other third party material in this article are included in the article's Creative Commons licence, unless indicated otherwise in a credit line to the material. If material is not included in the article's Creative Commons licence and your intended use is not permitted by statutory regulation or exceeds the permitted use, you will need to obtain permission directly from the copyright holder. To view a copy of this licence, visit http://creativecommons.org/licenses/by/4.0/ The Creative Commons Public Domain Dedication waiver (http://creativecommons.org/publicdomain/zero/1.0/) applies to the data made available in this article, unless otherwise stated in a credit line to the data. 
Blood culture is crucial for bacteremia detection [7]. Previous studies showed that early time to positivity (TTP) of blood culture can serve as a poor indicator for patients with different kinds of bacteremia [1, 8-11]. However, few studies reported the correlation between TTP and clinical outcomes in P. aeruginosa bacteremia children, and the optimal TTP cut-off point remained unclear. Therefore, the aim of our study is to evaluate the optimal TTP cut-off point, explore the correlation between TTP and clinical outcomes, which may help clinicians to identify risk factors of $P$. aeruginosa bacteremia children and provide more effective treatment earlier.

\section{Methods}

\section{Study designs and participants}

Children's Hospital of Chongqing Medical University is a 1500-bed tertiary teaching hospital in Chongqing, China, ranked among the top three domestic children's hospitals (rank list: http://top100.imicams.ac.cn/home). We conducted a retrospective study at this facility. Patients with $P$. aeruginosa bacteremia from August 2014 to November 2018 were identified retrospectively. The inclusive criteria were as follows: (i) inpatients; (ii) age < 18 years; (iii) with $\geq$ one positive $P$. aeruginosa blood culture; (iv) with systemic inflammatory manifestations. The exclusive criteria included any of the following: (i) patients with incomplete medical records; (ii) patients who missed their TTPs; (iii) patients with polymicrobial bacteremia.

\section{Microbiological methods}

An approximately $3-5 \mathrm{ml}$ of venous blood $(\geq 0.5 \mathrm{~mL}$ for neonate) was inoculated into aerobic each BACTEC PLUS bottle and transported to the microbiological laboratory at any time of the day $(24 \mathrm{~h}$ a day, 7 days a week). Blood cultures were processed employing the Becton-Dickinson diagnostic systems, which automated measured bacterial growth by continuously monitoring $\mathrm{CO}_{2}$ production in every $5 \mathrm{~min}$, through a fluorescent sensor technology. Those positive cultures were subsequently subcultured after Gram staining. The Vitek identification and susceptibility cards (bioMe'rieux Vitek) took charge of species identification and susceptibility detection.

\section{Definition}

$P$. aeruginosa bacteremia was defined as at least one blood culture positive for $P$. aeruginosa [9]. Time to positivity (TTP) was measured as the length of time span between the beginning of blood incubation and the alert signal by an automated system [11]. We only enrolled the shortest TTP if there were more than one positive sample. The immunosuppression was defined as primary immunodeficiency disease and/or receipt of high dose steroid therapy regularly more than half a month ( $\geq$ prednisolone $10 \mathrm{mg} /$ daily or equivalent dose), and/or receipt immunosuppressive chemotherapy within the last 2 months [1]. Neutropenia was defined as the number of neutrophils less than 500/l [12]. Nosocomial infection was defined when manifestations and positive blood culture were obtained more than $48 \mathrm{~h}$ after admission [12]. Pittsburgh bacteremia scoring system was used to evaluate the severity of bacteremia in children. We calculated the scores within 2 days prior or on the day of the first blood culture $[8,13,14]$, The source of infection was determined only when there were both clinical and laboratory evidence of the site on the day of the first blood culture [8]. Otherwise it was defined as primary bacteremia [12]. Metastatic foci of infection was defined as infection foci developed at least 2 days after the first positive blood culture with microbiologically or clinically evidence documented [15]. Appropriate antimicrobial therapy referred to receipt of at least one active intravenous antimicrobial agent according to susceptibility result within $24 \mathrm{~h}$ after blood samples were collected and before susceptibility results were available [16], otherwise it was defined as inappropriate antimicrobial therapy. MDR (multi-drug resistance) was defined as acquired nonsusceptibility to at least one agent in three or more antimicrobial categories [16]. Pseudomonas meningitis was diagnosed when patients fulfilled the following criteria: a positive $P$. aeruginosa culture of cerebrospinal fluid (CSF) and clinical evidence of $P$. aeruginosa meningitis [17]. P. aeruginosa peritonitis was diagnosed when patients had clinical evidence of an intraabdominal source of infection and a positive ascitic fluid culture with $P$. aeruginosa [18]. Septic shock was defined as patients with sepsis who need vasopressor to maintain a mean arterial pressure of $65 \mathrm{mmHg}$ or greater and serum lactate level greater than $2 \mathrm{mmol} / \mathrm{L}$ without hypovolemia [19]. Pneumonia was defined as clinical symptoms and signs in combination with radiologic evidence [20]. Hypoalbuminemia was defined as serum albumin concentration less than $2.5 \mathrm{~g} / \mathrm{dL}$ [21].

\section{Data collection}

Data retrieved from the medical records included demographic characteristics (age, sex, weight), underlying conditions, underlying diseases, place of bacteremia acquisition, the inappropriateness of empirical antibiotics use, TTP of blood culture, severity of bacteremia assessed by Pitt bacteremia scores and clinical outcomes.

\section{Outcomes assessment}

The primary outcome was in-hospital mortality. The second outcome was the incidence of septic shock. 


\section{Statistical analysis}

Classification variables were presented as numbers (n) and percentages (\%), and differences in proportions were compared by chi-squared test and Fisher's exact test if necessary. Continuous variables with abnormal distributions, presented as medians with inter-quartile ranges (IQRs), were analyzed by using the Mann Whitney $U$ test. Receiver-operating characteristic (ROC) analysis was conducted to determine the optimum cutoff point for TTP, with the maximum Youden's index was applied as the possible applicable predictive marker [20]. The predictive capability of TTP was assessed by the area under the ROC curve (AUC). $0.5<\mathrm{AUC} \leq 0.7$ implicated less predictive, $0.7<\mathrm{AUC} \leq 0.9$ indicated moderately predictive and $0.9<$ AUC $<1$ referred to highly predictive [22]. Univariate and multivariate logistic regression was employed to find the association between risk factors and in-hospital mortality, septic shock. Variables with $P$-value $<0.10$ in univariate analysis further evaluated in multivariate models with forward LR selection. Meanwhile, the variables with $P$-value $\leq 0.05$ were retained. Odds ratio (OR) and corresponding 95\% confidence interval (CI) was calculated. Hazard curves were further assessed by Kaplan-Meier method. All analyses were performed by using SPSS software for Unix (Version 23.0; SPSS, Chicago, IL, USA). A $P$-value < 0.05 (two-sided) was considered significant.

\section{Results}

Study population and patient characteristics

Sixty inpatients with $\geq$ one $P$. aeruginosa blood culture positive were enrolled retrospectively during this study period. Eleven of them were excluded (five cases had incomplete information, five cases were infected with other bacteria, one case missed his TTP). Therefore, 52 cases were analyzed in this study finally.

Median age of these patients was $1.79(0.43-9.0)$ years. Median weight was $11(7.00-27.00) \mathrm{kg}$, and the male account for $61.5 \%(32 / 52)$. The average of overall hospitalization stay was $22.52(9.05-38.3)$ days. The most common underlying conditions were immunosuppression $(50.0 \%, 26 / 52)$, followed by neutropenia $(46.2 \%$, $24 / 52)$ and hypoalbuminemia $(42.3 \%, 22 / 52)$. The most common underlying disease was hematologic malignancies $(16 / 52,30.8 \%)$, followed by congenital heart disease $(6 / 52,11.6 \%)$. The common complications were pneumonia $(17.3 \%, 9 / 52)$, meningitis $(9.6 \%, 5 / 52)$. The primary origins of infection were respiratory tract infection (42.3\%, 22/52), skin and soft tissue infection $(15.4 \%, 8 /$ $52)$, vascular-catheter related infection $(15.4 \%, 8 / 52)$, and primary infection $(13.5 \%, 7 / 52)$. Twenty $(38.5 \%)$ patients were admitted to intensive care unit. Twenty-two (42.3\%) patients were nosocomial. The median of Pitt bacteremia scores was 1.5 (1-4.00). Thirty-one (59.5\%)

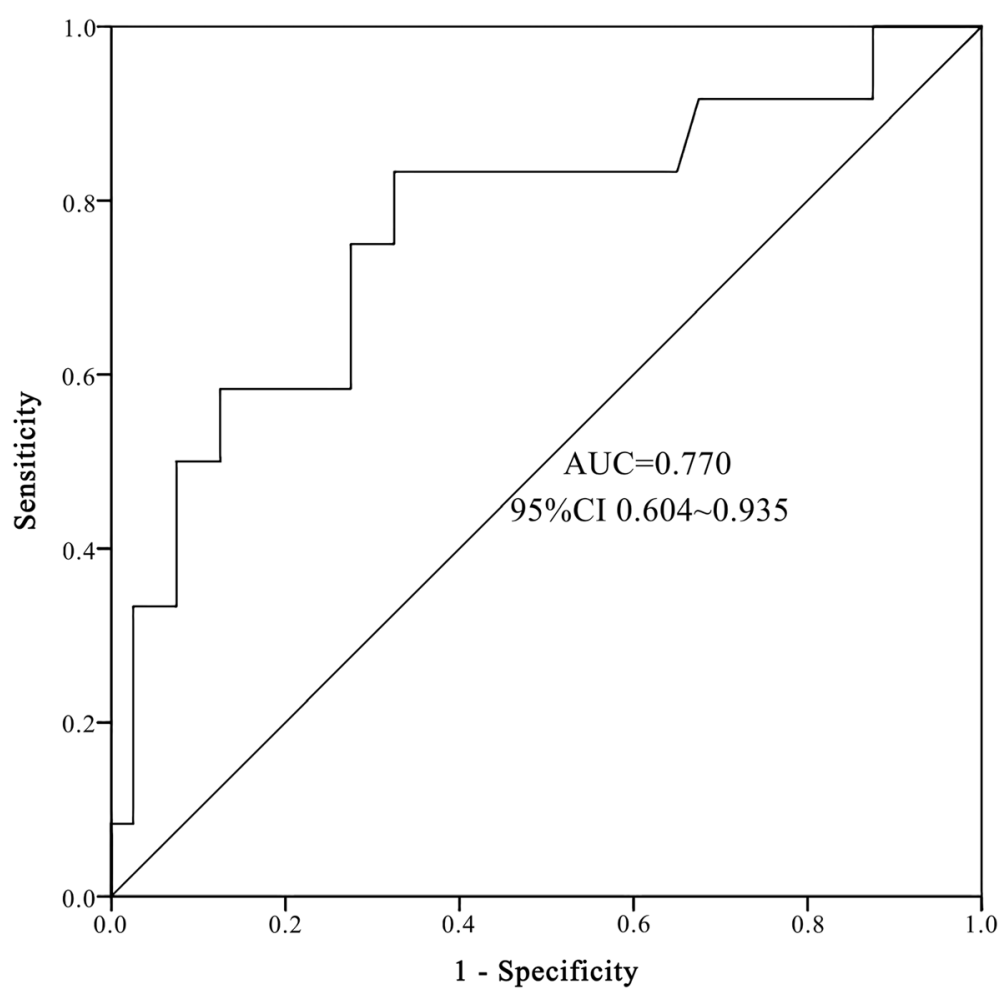

Fig. 1 ROC (receiver operating characteristic) curves of TTP (Time-to-positivity). AUC stands for area under the curve 
patients were given antibiotic prior to the blood culture, while $14(26.9 \%, 14 / 31)$ patients had received inappropriate empirically antimicrobial therapy. Four $(7.7 \%$, 4/ 52) patients were detected with MDR bacteria. The inhospital mortality was $23.1 \%(12 / 52)$, septic shock incidence was $28.8 \%$ (15/52). More details of clinical characteristics are shown in Table 1.

\section{TTP of $P$. aeruginosa bacteremia in children}

Median TTP was 18.74h (IQR 16.14-20.77). The optimal cut-off of TTP was evaluated by ROC analysis. The optimal point for TTP was $17.87 \mathrm{~h}$ with $75.0 \%$ sensitivity

Table 1 Clinical characteristics of 52 children with $P$. aeruginosa bacteremia

\begin{tabular}{|c|c|c|}
\hline Characteristics & $\mathrm{n} /$ median & $\% / \mathrm{IQR}$ \\
\hline \multicolumn{3}{|l|}{ Basic information } \\
\hline Age(years) & 1.79 & $0.43-9.0$ \\
\hline Male & 32 & 61.5 \\
\hline Weight(kilogram) & 11 & $7-27.00$ \\
\hline \multicolumn{3}{|l|}{ Underlying diseases } \\
\hline Hematologic malignancies & 16 & 30.8 \\
\hline Congenital heart disease & 6 & 11.6 \\
\hline \multicolumn{3}{|l|}{ Underlying conditions } \\
\hline Immunosuppression & 26 & 50.0 \\
\hline Neutropenia & 24 & 46.2 \\
\hline Hypoalbuminemia & 22 & 42.3 \\
\hline \multicolumn{3}{|l|}{ Complications } \\
\hline Pneumonia & 9 & 17.3 \\
\hline Meningitis & 5 & 9.6 \\
\hline Peritonitis & 3 & 5.8 \\
\hline \multicolumn{3}{|l|}{ Origins of infection } \\
\hline Respiratory tract infection & 22 & 42.3 \\
\hline Skin and soft tissue infection & 8 & 15.4 \\
\hline Vascular-catheter related infection & 8 & 15.4 \\
\hline Primary bacteremia & 7 & 13.5 \\
\hline Gastrointestinal infection & 5 & 9.6 \\
\hline Post-surgery or-procedure bacteremia & 2 & 3.8 \\
\hline Intensive unit care & 20 & 38.5 \\
\hline Nosocomial infection & 22 & 42.3 \\
\hline Pitts bacteremia scores & 1.5 & $1-4.00$ \\
\hline Antibiotics administration before blood culture & 31 & 59.5 \\
\hline TTP & 18.74 & 16.14-20.77 \\
\hline Length of hospitalization days & 22.52 & $9.05-38.3$ \\
\hline Multi-drug resistance bacteria & 4 & 7.7 \\
\hline \multicolumn{3}{|l|}{ Outcomes } \\
\hline Septic shock & 15 & 28.8 \\
\hline In-hospital mortality & 12 & 23.1 \\
\hline
\end{tabular}

and $72.5 \%$ specificity (AUC $0.77,95 \%$ CI $0.604-0.935$ ), indicating a moderate predicting capability (Fig. 1). Therefore $18 \mathrm{~h}$ was selected as the standard cut-off. The cases were divided into early TTP (TTP $\leq 18 \mathrm{~h}$ ) and late TTP group (TTP $>18 \mathrm{~h}$ ). The Kaplan-Meier survival curves of patients with the two TTP groups were shown in Fig. 2 and Fig. 3.

\section{Comparison of clinical characteristics between early and late TTP groups}

Table 2 shows the characteristics of early and late TTP groups. Early TTP group patients had significant higher in-hospital mortality ( $42.9 \%$ vs $9.7 \%, P=0.014$ ), higher incidence of septic shock $(52.4 \%$ vs $12.9 \%, P=0.006)$, higher Pitt bacteremia scores (3.00 vs $1.00, P=0.046)$ and more intensive care unit admission $(61.9 \%$ vs $22.6 \%$, $P=0.008$ ). There were more immunosuppression patients in late TTP group as compared to early TTP group (64.5\% vs $28.6 \%, P=0.023$ ). Four MDR bacteria were all detected in late TTP group patients. The demographic characteristics, underlying conditions, underlying diseases, the complications, origins of infection, nosocomial infection, antibiotics administration before blood culture, and length of hospitalization stay were with no remarkable differences (Table 2).

\section{Comparison of clinical characteristics between the survival and the non-survival groups}

The median TTP in non-survival group was 15.19 (IQR 11.21-18.24) hours, shorter than 19.42 (IQR 16.9220.97 ) hours in survival group $(P=0.005)$. Pitt scores in non-survival group were 4.50 (IQR 1.25-7.25), significantly higher than that in survival group [1.00 (IQR 1.00-3.75)]. The incidence of septic shock was remarkably higher in non-survival group when compared to survival group patients ( $58.3 \%$ vs $20.0 \%, P=0.025)$. More patients had hypoalbuminemia among fatal group than survival group $(75.0 \%$ vs $32.5 \%, P=0.023)$. No significant differences were detected in other clinical characteristics (Table 3).

\section{Risk factors of in-hospital mortality}

Univariate analysis revealed that early TTP, Pitt bacteremia scores $\geq 4$ and hypoalbuminemia were associated with inhospital mortality. Multivariate analysis showed early TTP (OR 5.88; 95\%CI 1.21-21.96) and Pitt bacteremia scores $\geq 4$ (OR 4.95; 95\%CI 1.26-27.50) were independently correlated with in-hospital mortality (Table 4).

\section{Risk factors of septic shock}

Univariate analysis also indicated that early TTP, Pitt bacteremia scores $\geq 4$, hypoalbuminemia and intensive care unit admission were correlated with septic shock. Multivariate analysis showed early TTP (OR 6.30; 95\%CI 


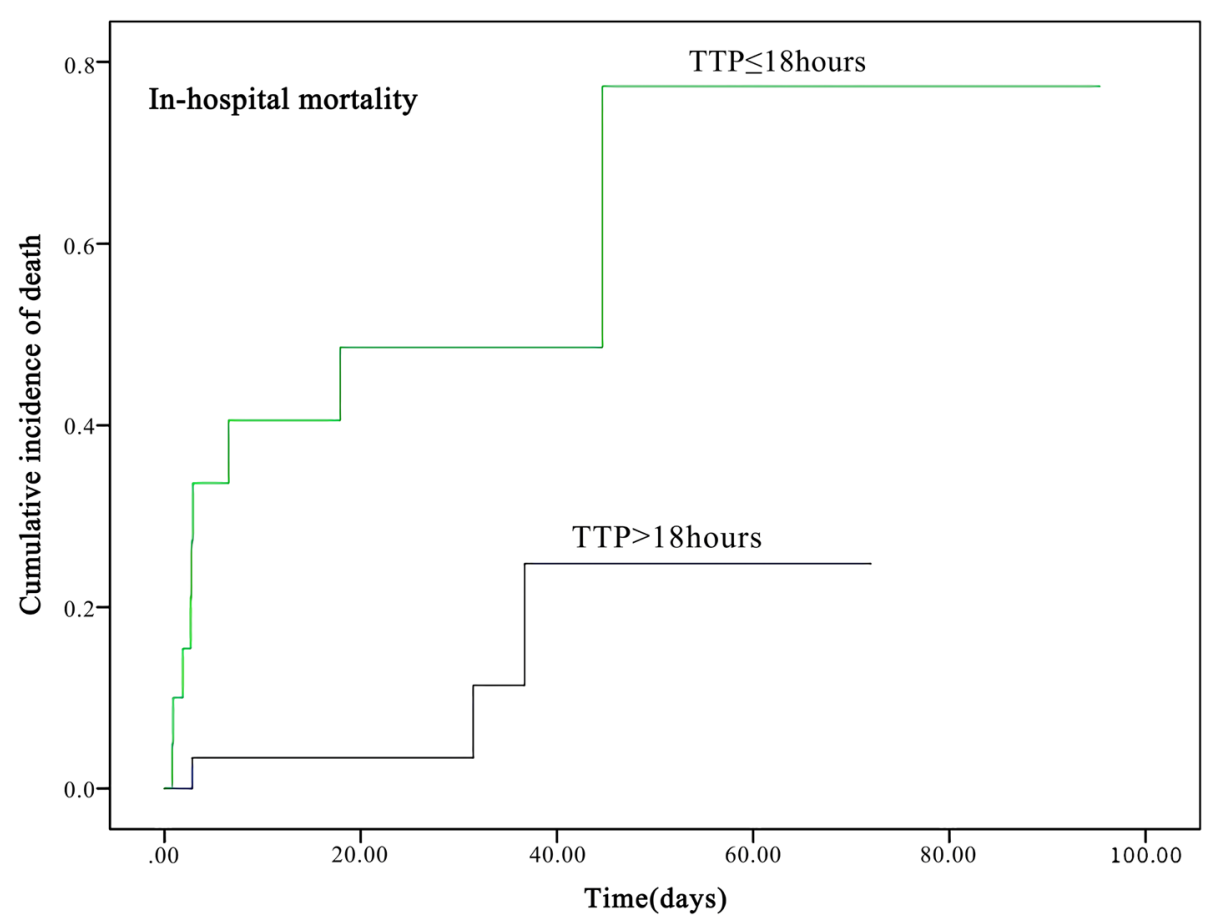

Fig. 2 The Kaplan-Meier survival curve of 52 children with P. aeruginosa bacteremia according to in-hospital mortality. Patients were divided into early and late TTP groups according to the optimal TTP cut-off points

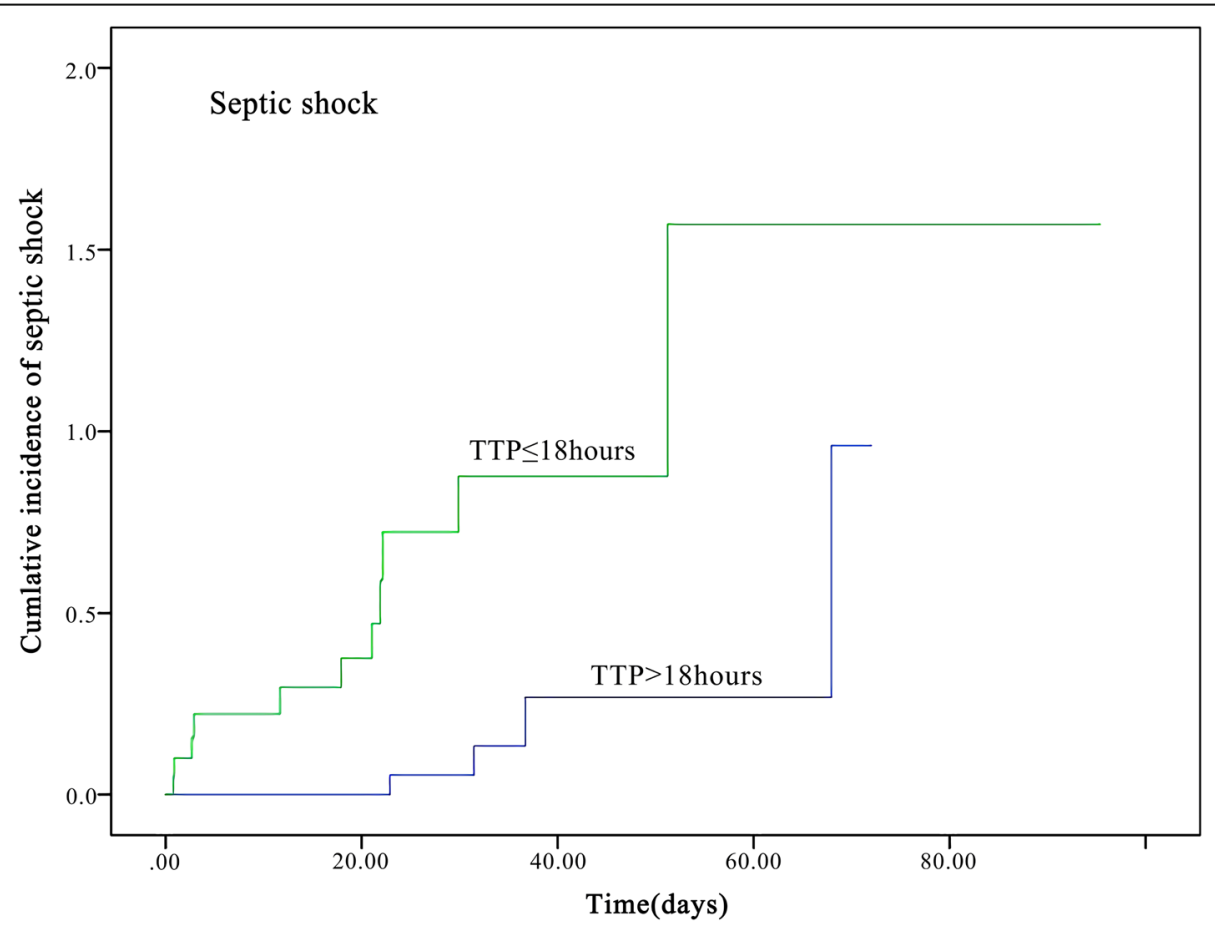

Fig. 3 The Kaplan-Meier survival curve of 52 children with $P$. aeruginosa bacteremia according to septic shock incidence. Patients were divided into early and late TTP groups according to the optimal TTP cut-off points 
Table 2 Clinical characteristics and outcomes associated with TTP in 52 children with P. aeruginosa bacteremia

\begin{tabular}{|c|c|c|c|}
\hline Characteristics & $\begin{array}{l}\text { Early TTP } \\
(T T P \leq 18 h, n=21)\end{array}$ & $\begin{array}{l}\text { Late TTP } \\
(T T P>18 h, n=31)\end{array}$ & $P$ values \\
\hline \multicolumn{4}{|l|}{ Basic information } \\
\hline Age(years) (median, IQR) & $0.85(0.48-8.79)$ & $2.66(0.30-9.01)$ & 0.911 \\
\hline Male (n, \%) & $11(52.4 \%)$ & $21(67.7 \%)$ & 0.384 \\
\hline Weight(kilogram)(median, IQR) & $8.5(7.5-24.75)$ & $13.5(6.00-28.00)$ & 0.668 \\
\hline \multicolumn{4}{|l|}{ Underlying diseases } \\
\hline Hematologic malignancies ( $n, \%)$ & $3(14.3 \%)$ & 13(41.9\%) & 0.070 \\
\hline Congenital heart disease $(n, \%)$ & $3(14.3 \%)$ & $3(9.7 \%)$ & 0.946 \\
\hline \multicolumn{4}{|l|}{ Underlying conditions } \\
\hline Immunosuppression (n, \%) & $6(28.6 \%)$ & $20(64.5 \%)$ & $0.023^{*}$ \\
\hline Neutropenia (n, \%) & $10(47.6 \%)$ & $14(45.2 \%)$ & 1.000 \\
\hline Hypoalbuminemia (n, \%) & 12(57.1\%) & 10(32.3\%) & 0.093 \\
\hline \multicolumn{4}{|l|}{ Complications } \\
\hline Pneumonia (n, \%) & $4(19.0 \%)$ & $5(16.1 \%)$ & 1.000 \\
\hline Meningitis ( $n, \%)$ & $3(14.3 \%)$ & $2(6.5 \%)$ & 0.645 \\
\hline Peritonitis & $2(9.5 \%)$ & $1(3.2 \%)$ & 0.727 \\
\hline \multicolumn{4}{|l|}{ Origins of infection } \\
\hline Respiratory tract $(\mathrm{n}, \%)$ & $11(52.4 \%)$ & $11(35.5 \%)$ & 0.263 \\
\hline Primary bacteremia (n, \%) & $2(9.5 \%)$ & $5(16.1 \%)$ & 0.787 \\
\hline Vascular-catheter related infection (n, \%) & $3(14.3 \%)$ & $5(16.7 \%)$ & 1.000 \\
\hline Soft tissue infection (n, \%) & $2(9.5 \%)$ & $6(19.4 \%)$ & 0.567 \\
\hline Gastrointestinal infection (n, \%) & $3(14.3 \%)$ & $2(6.5 \%)$ & 0.645 \\
\hline Post-surgery or-procedure bacteremia (n, \%) & $0(0.0 \%)$ & $2(6.5 \%)$ & 0.240 \\
\hline Intensive unit care (n, \%) & $13(61.9 \%)$ & $7(22.6 \%)$ & $0.008^{*}$ \\
\hline Nosocomial infection $(\mathrm{n}, \%)$ & $7(33.3 \%)$ & $15(48.4 \%)$ & 0.392 \\
\hline Pittsburgh bacteremia scores (median, IQR) & $3.00(1.00-5.00)$ & $1.00(1.00-4.00)$ & $0.046^{*}$ \\
\hline Antibiotics administration before blood culture $(n, \%)$ & $12(57.1 \%)$ & 19(61.3\%) & 0.781 \\
\hline Length of hospitalization days (median, IQR) & 21.04(2.82-41.29) & 28.92(11.92-36.71) & 0.176 \\
\hline Multi-drug resistance bacteria $(\mathrm{n}, \%)$ & $0(0.0 \%)$ & $4(12.9 \%)$ & 0.090 \\
\hline \multicolumn{4}{|l|}{ Outcomes } \\
\hline Septic shock (n, \%) & $11(52.4 \%)$ & $4(12.9 \%)$ & $0.006^{*}$ \\
\hline In-hospital mortality (n, \%) & $9(42.9 \%)$ & $3(9.7 \%)$ & $0.014^{*}$ \\
\hline
\end{tabular}

* Indicates statistical significant results, $P<0.05$

$1.18-33.77$ ), Pitt bacteremia scores $\geq 4$ (OR 8.15 ; $95 \%$ CI 1.53-43.32), hypoalbuminemia (OR 6.46; 95\% CI 1.1933.19) were independently associated with septic shock (Table 5).

\section{Discussion}

Studies showed that early TTP can serve as an indicator of higher bacterial burden in the blood $[1,13,16]$, which can be translated as more severe bacteremia. Different bacteria has different median TTP [23]. Several factors can influence TTP of blood culture, such as bacterial burden, blood volume of the sample, source of infection, use of antimicrobial agents and other clinical characteristics $[1,24]$. In this study, we found the optimal TTP cut-off point was $18 \mathrm{~h}$ in $P$. aeruginosa bacteremia children, which was significantly longer than that in adult $P$. aeruginosa bacteremia patients $(13 \mathrm{~h})$ [8]. The possible explanation could be that lower volume of blood culture of children compared to adults $(\geq 0.5 \mathrm{~mL}$ for neonate, $3-5 \mathrm{~mL}$ for children $\geq 1$ month [25], 8-10 $\mathrm{mL}$ for adults [26]) led to lower bacterial burden. Studies demonstrated that early TTP had significantly higher mortality in bacteremia patients caused by Grampositive bacteria such as S. pneumoniae [11], S. aureus [12], and Gram-negative bacteria such as E. coli [9], Klebsiella pneumoniae [10] and $P$. aeruginosa $[1,8]$ in 
Table 3 Comparison of clinical characteristics in survival and non-survival groups in 52 children with $P$. aeruginosa bacteremia

\begin{tabular}{|c|c|c|c|}
\hline Characteristics & $\begin{array}{l}\text { Non-survival } \\
(n=12)\end{array}$ & $\begin{array}{l}\text { Survival } \\
(n=40)\end{array}$ & $P$ values \\
\hline \multicolumn{4}{|l|}{ Basic information } \\
\hline Age(years) (median, IQR) & $1.55(0.21-9.80)$ & $1.79(0.44-9.01)$ & 0.373 \\
\hline Male (n, \%) & $8(66.7 \%)$ & $24(60.0 \%)$ & 0.938 \\
\hline Weight (kilogram) (median, IQR) & $10.00(4.85-29.88)$ & $11.25(7.00-27.00)$ & 0.521 \\
\hline \multicolumn{4}{|l|}{ Underlying diseases } \\
\hline Hematologic malignancies ( $n, \%)$ & $2(16.7 \%)$ & 14(35.0\%) & 0.395 \\
\hline Congenital heart disease $(n, \%)$ & $1(8.3 \%)$ & $6(12.5 \%)$ & 1.000 \\
\hline \multicolumn{4}{|l|}{ Underlying conditions } \\
\hline Immunosuppression (n, \%) & $5(41.7 \%)$ & $21(52.5 \%)$ & 0.743 \\
\hline Neutropenia (n, \%) & $8(66.7 \%)$ & $16(40.0 \%)$ & 0.195 \\
\hline Hypoalbuminemia (n, \%) & $9(75.0 \%)$ & $13(32.5 \%)$ & $0.023 *$ \\
\hline \multicolumn{4}{|l|}{ Complications } \\
\hline Pneumonia (n, \%) & $3(25.0 \%)$ & $6(15.0 \%)$ & 0.129 \\
\hline Meningitis ( $n, \%)$ & $2(16.7 \%)$ & $3(7.5 \%)$ & 0.699 \\
\hline Peritonitis (n, \%) & $0(0.0 \%)$ & $3(7.5 \%)$ & 0.333 \\
\hline \multicolumn{4}{|l|}{ Origins of infection } \\
\hline Respiratory tract $(\mathrm{n}, \%)$ & $6(50.0 \%)$ & $16(40.0 \%)$ & 0.740 \\
\hline Primary bacteremia (n, \%) & $1(8.3 \%)$ & $6(15.0 \%)$ & 0.911 \\
\hline Vascular-catheter related infection (n, \%) & $1(8.3 \%)$ & $7(17.5 \%)$ & 0.752 \\
\hline Soft tissue infection (n, \%) & $3(25.3 \%)$ & $5(12.5 \%)$ & 0.551 \\
\hline Gastrointestinal infection (n, \%) & $1(8.3 \%)$ & $4(10.0 \%)$ & 1.000 \\
\hline Post-surgery or-procedure bacteremia (n, \%) & $0(0.0 \%)$ & $2(5.0 \%)$ & 0.434 \\
\hline Intensive unit care (n, \%) & $6(50.0 \%)$ & 14(35.0\%) & 0.500 \\
\hline Nosocomial infection $(\mathrm{n}, \%)$ & $4(33.3 \%)$ & 18(45.0\%) & 0.701 \\
\hline Pittsburgh bacteremia scores (median, IQR) & $4.50(1.25-7.25)$ & $1.00(1.00-3.75)$ & $0.043^{*}$ \\
\hline Antibiotics administration before blood culture $(n, \%)$ & $7(58.3 \%)$ & $24(60.0 \%)$ & 1.000 \\
\hline Inappropriate empirical antimicrobial therapy (n, \%) & $5(41.7 \%)$ & $9(22.5 \%)$ & 0.267 \\
\hline TTP (median, IQR) & $15.19(11.21-18.24)$ & 19.42(16.92-20.97) & $0.005^{*}$ \\
\hline Length of hospitalization days (median, IQR) & $2.86(2.07-28.08)$ & $26.44(19.80-44.63)$ & $0.002^{*}$ \\
\hline Multi-drug resistance bacteria $(\mathrm{n}, \%)$ & $0(0.0 \%)$ & $4(10.0 \%)$ & 0.259 \\
\hline Septic shock (n, \%) & $7(58.3 \%)$ & $8(20.0 \%)$ & $0.025^{*}$ \\
\hline
\end{tabular}

* Indicates statistical significant results, $P<0.05$

Table 4 Logistic regression analysis of risk factors of in-hospital mortality among 52 children with $P$. aeruginosa bacteremia

\begin{tabular}{|c|c|c|c|c|c|c|}
\hline \multirow[t]{2}{*}{ Variables } & \multicolumn{3}{|c|}{ Univariate analysis } & \multicolumn{3}{|c|}{ Multivariate analysis } \\
\hline & $\mathrm{OR}$ & $95 \% \mathrm{Cl}$ & $P$ value & $\mathrm{OR}$ & $95 \% \mathrm{Cl}$ & $P$ value \\
\hline $\mathrm{TTP} \leq 18 \mathrm{~h}$ & 7.00 & $1.61-30.48$ & $0.001^{*}$ & 5.88 & $1.21-21.96$ & $0.035^{*}$ \\
\hline Pittsburgh bacteremia scores $\geq 4$ & 6.00 & $1.48-24.27$ & $0.012^{*}$ & 4.95 & $1.26-27.50$ & $0.024^{*}$ \\
\hline Hypoalbuminemia & 6.23 & $1.44-26.95$ & $0.014^{*}$ & & & \\
\hline Inappropriate empirical antimicrobial therapy & 2.46 & $0.63-9.65$ & 0.197 & & & \\
\hline Intensive care unit admission & 1.86 & $0.50-6.85$ & 0.352 & & & \\
\hline
\end{tabular}

* Indicates statistical significant results, $P<0.05$ 
Table 5 Logistic regression analysis of risk factors of septic shock among 52 children with $P$. aeruginosa bacteremia

\begin{tabular}{|c|c|c|c|c|c|c|}
\hline \multirow[t]{2}{*}{ Variables } & \multicolumn{3}{|c|}{ Univariate analysis } & \multicolumn{3}{|c|}{$\underline{\text { Multivariate analysis }}$} \\
\hline & $\mathrm{OR}$ & $95 \% \mathrm{Cl}$ & $P$ value & $\mathrm{OR}$ & $95 \% \mathrm{Cl}$ & $P$ value \\
\hline $\mathrm{TTP} \leq 18 \mathrm{~h}$ & 7.43 & $1.92-28.79$ & $0.004^{*}$ & 6.30 & $1.18-33.77$ & $0.032^{*}$ \\
\hline Pittsburgh bacteremia scores $\geq 4$ & 11.79 & $2.88-48.25$ & $0.001^{*}$ & 8.15 & $1.53-43.32$ & $0.014^{*}$ \\
\hline Hypoalbuminemia & 10.80 & $2.51-46.43$ & $0.001^{*}$ & 6.46 & $1.19-33.19$ & $0.031^{*}$ \\
\hline Inappropriate empirical antimicrobial therapy & 0.98 & $0.25-3.81$ & 0.979 & & & \\
\hline Intensive care unit admission & 8.56 & $2.18-33.63$ & $0.002^{*}$ & & & \\
\hline
\end{tabular}

* Indicates statistical significant results, $P<0.05$

adult bacteremia patients. Our previous studies indicated early TTP were associated with the worse outcomes in S. pneumoniae bacteremia children [27], Here, we found that TTP $\leq 18 \mathrm{~h}$ had moderate capability to predict inhospital mortality in $P$. aeruginosa bacteremia children $($ AUC $=0.770)$. Early TTP patients had approximately 5 folds higher risk of in-hospital mortality and 6 folds higher risk of septic shock when compared to late TTP. Our study indicated the association between early TTP and clinical outcomes in children with $P$. aeruginosa bacteremia, which was in accord with previous studies.

It is commonly accepted that Pitt bacteremia scores can evaluate severity of bacteremia and provide prognostic information, Pitt bacteremia scores $\geq 4$ can be assumed as critical bacteremia [8, 13, 14]. Previous study showed revealed that adult patients with Pitt bacteremia scores $\geq 4$ had approximately 13 folds higher risk of 30 days mortality [8]. Our study indicated that Pitt bacteremia scores was statistically higher in early TTP and fatal group respectively compared with late TTP and surviving group, which was in line with previous study. Moreover, multivariate analysis demonstrated that Pitt bacteremia scores $\geq 4$ was the independent risk factor of in-hospital mortality and septic shock.

We found 38 patients $(38 / 52,73.1 \%)$ received appropriate empirical antimicrobial therapy after blood culture before susceptibility results (23 patients had Carbapenems, 8 patients had Piperacillin-tazobactam, 6 patients had Cefepime, and 1 patient had Ceftazidime. Fourteen patients (26.9\%) received inappropriate empirical antimicrobial therapy after blood culture before susceptibility results (Cefotiam, Cefuroxime, Vancomycin, Teicoplanin, Latamoxef and Cefazolin sodium pentahydrate). Studies showed that receiving appropriate empirical antimicrobial agents earlier was significantly important $[1,28]$. While we showed no correlation between inappropriate empirical antimicrobial treatment and in-hospital mortality, which was in accord with several studies [14, 29]. The plausible explanations for the differences are as follows: we included community-required infection patients, the initial treatment outside our hospital could be an interference factor. Second, we did not determine the precise delay time correlated with in-hospital mortality.
Our study also revealed that lower level of albuminemia is a possible independent risk factor of septic shock in P.aeruginosa bacteremia children, which was not noted in previous studies of adults. Lokesh K, et al. [21] showed that hypoalbuminemic patients had worse outcomes. Probably, critical illness and sepsis were the reasons for hypoalbuminemia, which could cause lower plasma colloid osmotic pressure and inadequate blood perfusion to vital organs [21, 30]. Nevertheless, the benefit of receiving albumin in adults or childhood critical patients remains unclear $[21,30]$. Prospective study may be needed to clarify the effects of albumin in critical bacteremia children.

Studies in adult patients indicated the origin of infection was correlated with TTP. For E. coli bacteremia, urinary tract and vascular catheter-related infections had longer TTP (> 7 h) [31]. While $S$. aureus bacteremia patients with intravascular-catheter infections had shorter TTP ( $\leq 12 \mathrm{~h})$ [1]. As for $K$. pneumoniae bacteremia, primary bacteremia patients had earlier TTP $(<7 \mathrm{~h})$ [10]. No correlation was found between TTP and origins of infection in this study. Primary infection and lack of bacterial culture might have resulted in bias.

This study had some limitations. Firstly, this is a retrospective study, therefore prospective studies can be needed to strengthen our conclusion. Secondly, the small population size may lead to heterogeneous results. Thirdly, this is a single-center study. The relatively small sample and single-center study may lead to type II errors and decrease the ability to obtain solid proof, therefore, further studies with multi-center and a larger sample size are needed to address this conclusion.

\section{Conclusion}

In conclusion, our study revealed that early TTP (TTP $\leq 18 \mathrm{~h}$ ), along with Pitt bacteremia scores $\geq 4$ could predict poor outcomes for children with $P$. aeruginosa bacteremia. Therefore, TTP can serve as a prognostic tool by clinicians.

\section{Abbreviations}

TTP: Time to positivity; P. aeruginosa: Pseudomonas aeruginosa; APACHE scores: Acute physiology and chronic health evaluation scores; PRISM scores: Pediatric risk of mortality scores; Cl: Confidence interval; OR: Odds 
ratio; ROC: Receiver operating characteristic; IQR: Inter-quartile range; CSF: Cerebrospinal fluid; MDR: Multi-drug resistance

\section{Acknowledgments}

We thank the many staff the Department of Respiratory Medicine, Children's Hospital of Chongqing Medical University.

\section{Authors' contributions}

$Z X L$ designed and revised the experiments; $H T X, J C, Q H Y$ and $G L Z$ collected and checked the date. HT X carried out the experiments and wrote the manuscript; QY, QY L and XY T contributed to drawing figures and tables; SY L, YY L and DP C helped in the statistical analyses; all authors contributed to manuscript revisions and approved the final version for publication.

\section{Funding}

This work was supported by national key clinical specialty(grant no.2011873).

\section{Availability of data and materials}

The data-sets analyzed during the current study are available from the corresponding author on reasonable request.

\section{Ethics approval and consent to participate}

The study was approved by the Ethics Committee of Children's Hospital of Chongqing Medical University (File No. (2019)304). Informed consent was obtained from the parents in written form when children were admitted to the hospital. All methods were performed in accordance with the relevant guidelines and regulations.

\section{Consent for publication}

Not applicable.

\section{Competing interests}

The authors declare no financial and non-financial competing interests.

\section{Author details}

${ }^{1}$ Key Laboratory of Pediatrics in Chongqing, Chongqing 400014, China. ${ }^{2}$ China International Science and Technology Cooperation base of Child development and Critical Disorders, Department of Children's Hospital of Chongqing Medical University of Education, Ministry of Education Key Laboratory of Child Development and Disorders, Chongqing 400014, China. ${ }^{3}$ Department of Respiratory Medicine, Children's Hospital of Chongqing Medical University, Chongqing 401122, China. ${ }^{4}$ Department of Clinical Laboratory center, Children's Hospital of Chongqing Medical University, Chongqing 400014, China.

Received: 17 October 2019 Accepted: 14 July 2020

Published online: 09 September 2020

\section{References}

1. Willmann M, Kuebart I, Vogel W, et al. Time to positivity as prognostic tool in patients with Pseudomonas aeruginosa bloodstream infection. J Inf Secur. 2013;67:416-23.

2. Endimiani A, Luzzaro F, Pini B, et al. Pseudomonas aeruginosa bloodstream infections: risk factors and treatment outcome related to expression of the PER-1 extended-spectrum beta-lactamase. BMC Infect Dis. 2006:6:52

3. Rojas A, et al. Rates, predictors and mortality of community-onset bloodstream infections due to Pseudomonas aeruginosa: systematic review and meta-analysis. Clin Microbiol Infect. 2019;25:964-70.

4. Lodise TP, Patel N, Kwa A, et al. Predictors of 30-day mortality among patients with Pseudomonas a eruginosa bloodstream infections: impact of delayed appropriate antibiotic selection. Antimicrob Agents Ch. 2007;51: 3510-5.

5. Tumbarello M, Repetto E, Trecarichi EM, et al. Multidrug-resistant Pseudomonas aeruginosa bloodstream infections: risk factors and mortality. Epidemiol Infect. 2011;139:1740-9.

6. Pai S, Enoch DA, Aliyu SH. Bacteremia in children: epidemiology, clinical diagnosis and antibiotic treatment. Expert Rev Anti-infe. 2015;13:1073-88.
7. Book M, Lehmann LE, Zhang XH, et al. Monitoring infection: from blood culture to polymerase chain reaction (PCR). Best Pract Res Cl Ob. 2013;27(2): 279-88.

8. Tang PC, Lee CC, Li CW, et al. Time-to-positivity of blood culture: an independent prognostic factor of monomicrobial Pseudomonas aeruginosa bacteremia. J Microbiol Immunol. 2017:50:486-93.

9. Peralta G, Roiz MP, Sánchez MB, et al. Time-to-positivity in patients with Escherichia coli bacteraemia. Clin Microbiol Infect. 2007;13:1077-82.

10. Liao CH, Lai CC, Hsu MS, et al. Correlation between time to positivity of blood cultures with clinical presentation and outcomes in patients with Klebsiella pneumoniae bacteraemia: prospective cohort study. Clin Microbiol Infect. 2009;15:1119-25.

11. Peralta G, Rodríguez-Lera MJ, Garrido JC, et al. Time to positivity in blood cultures of adults with $\mathrm{S}$ treptococcus pneumoniae bacteremia. BMC Infect Dis. 2006;6:79.

12. Marra AR, Edmond MB, Forbes BA, et al. Time to blood culture positivity as a predictor of clinical outcome of Staphylococcus aureus bloodstream infection. J Clin Microbiol. 2006;44:1342-6.

13. Haimi-Cohen Y, Vellozzi EM, Rubin LG. Initial concentration of Staphylococcus epidermidis in simulated pediatric blood cultures correlates with time to positive results with the automated, continuously monitored BACTEC blood culture system. J Clin Microbiol. 2002;40:898-901.

14. Lodise TP, Patel N, Kwa A, et al. Predictors of 30-day mortality among patients with Pseudomonas aeruginosa bloodstream infections: impact of delayed appropriate antibiotic selection. Antimicrob Agents Ch. 2007;51: 3510-5.

15. Bisbe J, Gatell JN, et al. Pseudomonas aeruginosa bacteremia: Univariate and multivariate analyses of factors influencing the prognosis in 133 episodes. Clin Infect Dis. 1988;10:629-35.

16. Morata L, Cobos-Trigueros N, Martínez JA, et al. Influence of multidrug resistance and appropriate empirical therapy on the 30-day mortality rate of Pseudomonas aeruginosa bacteremia. Antimicrob Agents Ch. 2012;56:4833-7.

17. Pai $S$, Bedford L, Ruramayi $R$, et al. Pseudomonas aeruginosa meningitis/ ventriculitis in a UK tertiary referral hospital. QJM: Int J Med. 2015;109:85-9.

18. Soriano G, Castellote J, Álvarez C, et al. Secondary bacterial peritonitis in cirrhosis: a retrospective study of clinical and analytical characteristics, diagnosis and management. J Hepatol. 2010;52:39-44.

19. Rhodes A, Evans LE, Alhazzani W, et al. Surviving sepsis campaign: international guidelines for management of sepsis and septic shock: 2016 Intens Care Med. 2017:43:304-77.

20. Bradley JS, Byington CL, Shah SS, et al. The management of communityacquired pneumonia in infants and children older than 3 months of age: clinical practice guidelines by the Pediatric Infectious Diseases Society and the Infectious Diseases Society of America. Clin Infect Dis. 2011;53:e25-76.

21. Tiwari LK, Singhi $S$, Jayashree $M$, et al. Hypoalbuminemia in critically sick children. Indian J Crit Care Med. 2014;18:565.

22. Faraggi D, Reiser B. Estimation of the area under the ROC curve. Stat Med. 2002;21:3093-106

23. Defrance G, Birgand G, Ruppé $\mathrm{E}$, et al. Time-to-positivity-based discrimination between Enterobacteriaceae, Pseudomonas aeruginosa and strictly anaerobic gram-negative bacilli in aerobic and anaerobic blood culture vials. J Microbiol Meth. 2013;93:77-9.

24. Yu VL, Chiou CCC, Feldman C, et al. An international prospective study of pneumococcal bacteremia: correlation with in vitro resistance, antibiotics administered, and clinical outcome. Clin Infect Dis. 2003:37:230-7.

25. Connell TG, Rele M, et al. How reliable is a negative blood culture result? Volume of blood submitted for culture in routine practice in a Children's hospital. Pediatrics. 2007;119:891-896.35.

26. Lin HH, Liu YF, Tien N, et al. Evaluation of the blood volume effect on the diagnosis of bacteremia in automated blood culture systems. J Microbiol Immunol. 2013;46:48-52

27. Li Q, Li Y, Yi Q, et al. Prognostic roles of time to positivity of blood culture in children with Streptococcus pneumoniae bacteremia. Eur J Clin Microbiol 2019; https://doi.org/https://doi.org/10.1007/s10096-018-03443-5.

28. Cunha BA. Sepsis and septic shock: selection of empiric antimicrobial therapy. Crit Care Clin. 2008:24:313-34.

29. Artero A, Zaragoza R, Camarena JJ, et al. Prognostic factors of mortality in patients with community-acquired bloodstream infection with severe sepsis and septic shock. J Crit Care. 2010;25:276-81.

30. SAFE Study investigators. A comparison of albumin and saline for fluid resuscitation in the intensive care unit. New Eng J Med. 2004;350:2247-56. 
31. Martinez JA, Soto S, et al. Relationship of phylogenetic background, biofilm production, and time to detection of growth in blood culture vials with clinical variables and prognosis associated with Escherichia coli bacteremia. J Clin Microbiol. 2006:44:1468-74.

\section{Publisher's Note}

Springer Nature remains neutral with regard to jurisdictional claims in published maps and institutional affiliations.

- fast, convenient online submission

- thorough peer review by experienced researchers in your field

- rapid publication on acceptance

- support for research data, including large and complex data types

- gold Open Access which fosters wider collaboration and increased citations

- maximum visibility for your research: over $100 \mathrm{M}$ website views per year

At $\mathrm{BMC}$, research is always in progress. 